
\title{
$\begin{array}{ll}\text { Research Square } & \text { Preprints are preliminary reports that have not undergone peer review. } \\ \text { They should not be considered conclusive, used to inform clinical practice, } \\ \text { or referenced by the media as validated information. }\end{array}$
}

\section{Weaning and Extubation Assisted with Conversational Hypnosis: A systematic review}

Johan Wormser ( $\sim$ jwormser@ghpsj.fr)

Groupe hospitalier Paris Saint-Joseph https://orcid.org/0000-0002-1542-9155

Christophe Romanet

Groupe hospitalier Paris Saint-Joseph

Aurel Bellaïche

Groupe hospitalier Paris Saint-Joseph

François Philippart

Groupe hospitalier Paris Saint-Joseph

\section{Research Article}

Keywords: weaning process, extubation, intensive care unit, hypnotherapy, dyspnea, anxiety

Posted Date: May 25th, 2021

DOI: https://doi.org/10.21203/rs.3.rs-273882/v2

License: @ (i) This work is licensed under a Creative Commons Attribution 4.0 International License. Read Full License 


\section{Abstract}

Background: Patients admitted in intensive care units often receive mechanical ventilation. Prior to extubation, weaning process could be a major cause of dyspnea and anxiety in awake intubated patients. Hypnosis is commonly used to manage anxiety and pain. Our hypothesis is that hypnosis would decrease stress during the weaning and extubation process.

Methods: This systematic review follows PRISMA Guidelines and was registered in PROSPERO (CRD42020171445). Web of Science, MEDLINE and Cochrane Central Register of Controlled Trials databases were searched until 11 March 2020. We included all interventional trials assessing hypnotherapy for intubated patients whom were undergoing weaning or extubation. Two of the authors independently screened studies, extracted data and assessed risk of bias from identified trials.

Results: One study fulfilled our selection. This case series included 2 patients. Eleven sessions were completed. Dyspnea was not assessed/reported. Hypnotherapy decreased anxiety during the weaning process from a median of 1 point (Interquartile Range: 1.0-2.5). Increase in oxygen saturation is also reported. There was no evidence of neither an anxiety reduction or an oxygen saturation improvement. This is due to the scarcity of publication and the methodological design of the one selected study.

Conclusions: We cannot conclude whether or not hypnosis could be recommended in the weaning process of intubated patients. Further studies are needed to assess hypnosis effects.

Registration: The present systematic review was registered in the International prospective register of systematic reviews (PROSPERO) under registration number CRD42020171445.

\section{Introduction}

During intensive care unit (ICU) hospitalization, life-threatening situations frequently need the use of specific critical care devices (mechanical ventilation, hemodialysis, syringe pumps, extracorporeal membrane oxygenation etc.) and drugs (sedation, neuromuscular blocking agents etc.) improving survival but associated with post-ICU psychological issues. Studies point out that survivors tend to remember their hospital stay as a traumatic event filled with stressors such as pain, thirst and the inability to speak or move [1-3]. Ventilatory support and the endotracheal tube were most often described as one of the most uncomfortable devices [1,2]. The use of the latter furthermore usually leading to an unpleasant feeling of drowning : dyspnea [4].

The ventilatory support is eventually removed when the acute disorder is treated. Ventilatory weaning requires to decrease the ventilatory support received by the patient from the mechanical ventilator. During this weaning process patients' awakeness is essential, needing a reduction in sedation [5]. However, this sedation decrease may lead to a discrepancy with the parallel increasing ventilatory effort and therefore dyspnea, anxiety and stress could result. In fact, previous publications reported an association between 
dyspnea, mechanical ventilation and the development of Post-Traumatic Stress Disorder (PTSD) symptoms [6].

Only few non pharmacological treatments are used to reduce stress factors in mechanically ventilated patients. In a Cochrane review, Bradt and Dileo suggested that music listening may improve anxiety in mechanically ventilated patients [7].

However, there is a growing interest for hypnotherapy in many health settings such as anaesthesia [8], chronics disorders [9] or during invasive procedures [10]. Hypnotherapy is indeed commonly used to manage pain and anxiety or reduce the need of medication. Recently, Anlló et al. found that a 15 minutes hypnosis session reduced anxiety, dyspnea and respiratory rate in stable COPD patients [11]. Finally, Rousseaux et al. published a protocol trial which aims to compare hypnosis alone or combined with virtual reality to improve anxiety, pain and fatigue in patients hospitalized in ICU departments after cardiac surgery [12].

To date, studies have seldom assessed the effect of hypnotherapy in critical care. For this reason, its role in mechanically ventilated patients still remains unclear.

The primary aim of this systematic review was to determine whether hypnotherapy during ventilatory weaning and extubation could improve dyspnea.

\section{Methods}

The PRISMA guidelines were used to report this review [13]. The present systematic review was registered in the International prospective register of systematic reviews (PROSPERO) under registration number CRD42020171445.

\section{Search Strategy}

We searched for eligible studies until 11 March 2020 in the following databases: Web of Science, MEDLINE, Cochrane Central Register of Controlled Trials (See Appendix 1 for the search strategy). We searched on the OpenGrey database and also reviewed references lists of retrieved studies.

\section{Eligibility Criteria}

We included all types of interventional trials. Only publications in English, French or Spanish were selected. Studies had to include participants whom were in Intensive Care Units (ICU), ventilated with an endotracheal tube, and whom were undergoing respiratory weaning or extubation. Only adults were selected.

We included trials evaluating hypnotherapy during mechanical ventilation weaning or extubating process. If the study included at least two groups, one of them would not have received hypnotherapy.

\section{Outcome measures}


The primary outcome was self-reported dyspnea, without any restriction on the method of measurement.

Secondary outcomes were self-reported anxiety, stress and pain without any restriction on the method of measurement; and extubation success, defined as the proportion of patients free from invasive ventilatory support 48 hours after extubation.

\section{Study Selection}

Two authors per trial (JW, AB) assessed the titles and abstracts from reference lists for possible inclusion. These review authors read the full text of articles to make the final decision for inclusion. Disagreements were resolved by discussion (JW, AB). When inclusion was still questionable even with the full text, another reviewer was contacted (CR) to determine whether the article met inclusion criteria. Duplicate publications of the same study were noted, but each trial was counted only once.

\section{Data Extraction and Quality Assessments}

Two review authors per trial (JW, $A B$ ) extracted the characteristics for each trial. The results were compared, disagreements resolved by discussion (JW, AB, CR). We extracted information on the trial design, number included/analysed, participants' characteristics, country of study, method of assessment, inclusion/exclusion criteria, outcomes, follow-up times, adverse events, dropouts and withdrawals, intervention method and findings as they were reported in the paper.

Two review authors per trial (JW, AB) independently assessed risk of bias. For case report or case-series studies, the evaluation was based on the tool developed by Murad et al. for evaluating the methodological quality of this specific study design [14]. For case-control or cohort studies, the Newcastle Ottawa Scale was used [15]. For randomized and non-randomized controlled clinical trials (respectively RCT and CCT), the evaluation was based on the PEDro scale [16]. A study was considered as "high quality" if all the items were present, except those who evaluated the patient and therapist's blinding (items 5 and 6). If any other item was not found in the publication, the study was considered as "low quality".

We used the tool developed by van Tulder et al. for evaluating the level of evidence [17] (Table 1). 
Table 1

Level of Evidence
a. Strong - consistent findings amongst multiple high quality RCTs.
b. Moderate - consistent findings amongst multiple low quality RCTs and/or CCTs and/or one high quality RCT

c. Limited - one low quality RCT and/or CCT

d. Conflicting - inconsistent findings amongst multiple trials (RCT and/or CCTs)

e. No evidence from trials - no RCTs or CCTs

Findings were considered as consistent if all studies had the same conclusions.

Abbreviations : RCT: Randomized Controlled Trials; CCT: Nonrandomized Controlled Trials

To minimize the effect of publication bias, we searched the grey literature. To reduce the reporting bias, we consulted CT.gov looking for described protocols of the included studies.

\section{Results}

\section{Study Selection and study characteristics}

Thirteen potential studies were retrieved through the search strategies and other hand searched sources. After removing duplicates, we had 10 records. Of these 10 records we included 4 based on the informations in the title and/or abstract. Of those abstracts, we excluded 3 records after reading the full text. Characteristics of excluded studies [18-20] are listed in Table 2. A flow chart summarizing the study selection process is shown in Fig. 1.

Table 2

Characteristics of excluded studies

\begin{tabular}{|ll|}
\hline Excluded study & Reasons of exclusion \\
\hline Bowen DE. et al ${ }^{18}$ & Patient included was tracheotomized \\
LaRiccia P. et al. ${ }^{19}$ & Patient included was tracheotomized \\
Treggiari-Venzi M. et al. ${ }^{20}$ & Patient included was tracheotomized \\
\hline
\end{tabular}

We included 1 study [21]. The study's most important characteristics are summarized in Table 3. This trial was in English and had been conducted in the United States. This trial was designed as a case report. 
Table 3

Most important characteristics of the included study

Methods Study design: Case report

$N=(A /) 2 / 2$

Country: United States

Participants Inclusion criteria: two patients

- Patient 1: a 60-years-old female with past diagnosis of COPD and amyotrophic lateral sclerosis, hospitalized for a right-sided acoustic neuroma. She was placed on ventilator support post-surgery.

The patient was asked to consider the use of hypnotic relaxation to increase her weaning time after 22 days of mechanical ventilation. A written consent from the patient was obtained.

- Patient 2: a 78-years-old man with past medical history of pulmonary tuberculosis, alcoholism, chronic cough, and COPD, intubated after being found unconscious.

The patient agreed to try hypnosis relaxation 6 days after reintubation (attempted 11 days after the first intubation) and a written consent form was obtained from the patient and physician.

Exclusion criteria: none

Intervention Intervention: The preinduction procedure involved the education of the patient regarding the facts and myths of hypnosis. An eye-fixation method was used, trance stat was tested by the procedure described by Friedlander and Sarbin (1938). This involves suggesting a series of motor exercises. The trance lasted approximately 20 minutes. Both patients were seated in a comfortable chair, ear oximetry was monitored before, during and after hypnosis.

The first patient was unable to perform the eye closure and catalepsy test because of the paralysis of her right ocular muscles.

Control: none

Outcomes - Anxiety, with a 4-point scale (0-calm, 1-mild anxiety, 2-moderate anxiety, 3-severeanxiety)

- Oximetry

Follow-up times: 8 days of hypnosis sessions and 5 days without hypnosis for the first patient. 3 days of hypnosis sessions and 9 days without hypnosis for the second patient.

Reported findings:

- Reduction of anxiety level (loss between 1 and 3 points after hypnosis session)

- Increase in oxygen saturation after each session

- No need for ventilatory support during the waking hours for the first patient

- Successful extubation for the second patient. 


\begin{tabular}{|ll|}
\hline Methods & Study design: Case report \\
& $\begin{array}{l}\mathrm{N}=(\mathrm{A} / \mathrm{l}) 2 / 2 \\
\text { Country: United States }\end{array}$ \\
\hline Notes & $\begin{array}{l}\text { Dropouts and withdrawals: none } \\
\text { Adverse events: none }\end{array}$ \\
\hline
\end{tabular}

\section{Participants Characteristics}

The selected study involved 2 patients. Participants' ages varied from 60 to 78 years, respectively a female and a male. These patients had substantial co-morbidities like chronic obstructive pulmonary disease (COPD) and amyotrophic lateral sclerosis (ALS) for one of them and COPD, alcoholism and tuberculosis for the other one. The interventions began respectively on the 13th and 23rd day of mechanical ventilation support.

\section{Risk of bias in included study}

In agreement with the tool developed by Murad et al. [14] only exposure, follow-up and reporting bias were potentially reduced. Concerning the other criterias identified, none of them were sufficiently described to be considered as fulfilled. Evaluation of each criteria is given in Table 4. 
Table 4

Risk of bias of the included study

\section{Risk of bias}

\begin{tabular}{|c|c|c|}
\hline Bias & $\begin{array}{l}\text { Authors' } \\
\text { judgement }\end{array}$ & Support for judgement \\
\hline Selection & No & $\begin{array}{l}\text { Only two patients described, context described but peculiar } \\
\text { patients. }\end{array}$ \\
\hline Exposure & Yes & Time per session recorded, description of the intervention. \\
\hline Outcome & No & $\begin{array}{l}\text { Anxiety defined, measured and reported. Success of the } \\
\text { weaning and measurement of oximetry are also reported but } \\
\text { the presented graphic does not allow any accurate measure } \\
\text { of the values. }\end{array}$ \\
\hline Alternative causes & No & $\begin{array}{l}\text { The weaning period before the intervention seems too short } \\
\text { to clearly recognise the effect of hypnosis. For patient } n^{\circ} 2 \text {, the } \\
\text { intervention seems too close to the reintubation. }\end{array}$ \\
\hline $\begin{array}{l}\text { Challenge/rechallenge } \\
\text { phenomenon }\end{array}$ & No & Hypnosis maintained during the entire process. \\
\hline Dose-response effect & No & Same intensity of treatment. \\
\hline Follow-up & Yes & $\begin{array}{l}\text { Patients left the hospital } 5 \text { and } 9 \text { days after the last session } \\
\text { without mechanical ventilation (during waking hours or at } \\
\text { all). }\end{array}$ \\
\hline Reporting & Yes & Intervention and results are clearly described. \\
\hline
\end{tabular}

\section{Intervention used in the trials}

Description of the hypnosis procedure followed in the one included trial is given in the Table 3.

\section{Outcomes reported}

Outcomes measured in this trial were anxiety (4-point scale before and after each session) and oxygen saturation.

\section{Effects of intervention on primary outcome}

Dyspnea was not reported.

\section{Effects of intervention on secondary outcome}

Eleven sessions were described in Acosta's study. The median decrease in anxiety reported was 1 point (IQR: 1.0-2.5). Every session, anxiety declined by at least one point; the maximum decrease reported was 3 points. Stress and pain were not reported.

Only one of the two patients of this study was extubated on the third day of hypnotherapy. The second patient was able to breathe without the need for ventilatory support during the waking hours after 8 days 
of intervention. Nonetheless, this patient was discharged (transferred) from the hospital 5 days after the last hypnosis session.

\section{Any other outcome reported by the trials}

The author reported an increase of oxygen saturation during and after the hypnosis session. No statistical analysis was performed and data in numeric form were not presented in the paper.

\section{Discussion}

Data published about hypnotherapy during the weaning process for intubated patients are scarce. In fact, only one study fulfilled our selection. This case series included two patients with a variety of comorbidities. Results showed decrease in anxiety from at least one point per hypnosis session. Oxygen saturation may have been increased, but no exploitable data were provided to objectify it.

According to the van Tulder et al. Tool [17], there is no evidence supporting the efficacy of hypnotherapy in the weaning process. This is due to the included study design (a case report). This highlights the need for further controlled studies.

A predefined protocol was recorded on PROSPERO in order to reduce the risk of bias in the review process. To minimize publication bias, we searched the gray literature and on clinical trial registration sites.

To our knowledge, there is to date no review investigating the same intervention or population of interest. However, 3 studies were excluded from this paper because hypnosis interventions were performed on tracheotomized patients [18-20] and hence not related to the extubation process. In two of these 3 cases series, reductions in anxiety were reported with hypnosis sessions. Nethertheless, anxiety assessment is not clearly described.

Even if those three studies' findings are consistent with Acosta's, designs used in all four studies make the data unusable for a proper statistical analysis.

Moreover, weaning tracheotomized patients and the extubation process are two specific nontransposable phenomenons, making the findings of those three previous studies unusable as well for our systematic review.

\section{Conclusion}

Trials specific to hypnotherapy use in ventilatory weaning or extubation are rare. We found only one publication with a poor study design. Due to this publication scarcity, we cannot conclude whether or not hypnotherapy should be recommended.

Due to the absence of evidence in controlled trials, further studies are needed to determine the actual effect of hypnotherapy. These randomized controlled studies should investigate if hypnotherapy is 
feasible during the weaning process and the extubation and how it impacts self-reported dyspnea, anxiety and pain and weaning success.

\section{Declarations}

\section{Ethics approval and consent to participate}

Not applicable for a systematic review.

\section{Consent for publication}

Not applicable for a systematic review.

\section{Availability of data and materials}

Original data is available from the corresponding author on reasonable request.

\section{Competing interests}

The authors have no conflicts of interest to declare.

\section{Funding}

This research did not receive any funding.

\section{Authors' contributions}

JW, CR, AB and FP conceived and planned the following research. JW, AB performed the literature search, study inclusion, data extraction and risk of bias assessment with support from CR for any disagreement. $\mathrm{JW}, \mathrm{CR}, \mathrm{AB}$ and FP wrote the manuscript, provided critical analysis of the manuscript redaction and final approval.

\section{References}

1. Khalaila R, Zbidat W, Anwar K, Bayya A, Linton DM, Sviri S. Communication difficulties and psychoemotional distress in patients receiving mechanical ventilation. Am J Crit Care nov. 2011;20(6):470-9.

2. Samuelson KAM. Adult intensive care patients' perception of endotracheal tube-related discomforts: a prospective evaluation. Heart Lung févr. 2011;40(1):49-55.

3. Gültekin Y, Özçelik Z, Akıncı SB, Yorgancı HK. Evaluation of stressors in intensive care units. Turk J Surg. 2018;34(1):5-8.

4. Schmidt M, Demoule A, Polito A, Porchet R, Aboab J, Siami S, et al. Dyspnea in mechanically ventilated critically ill patients. Crit Care Med sept. 2011;39(9):2059-65. 
5. Boles J-M, Bion J, Connors A, Herridge M, Marsh B, Melot C, et al. Weaning from mechanical ventilation. Eur Respir J mai. 2007;29(5):1033-56.

6. Worsham CM, Banzett RB, Schwartzstein RM. Dyspnea AR, Failure. Psychological Trauma, and PostICU Mental Health: A Caution and a Call for Research. Chest févr. 2021;159(2):749-56.

7. Bradt J, Dileo C. Music interventions for mechanically ventilated patients. Cochrane Database Syst Rev. 2014;(12):CD006902.

8. Rosendahl J, Koranyi S, Jacob D, Zech N, Hansen E. Efficacy of therapeutic suggestions under general anesthesia: a systematic review and meta-analysis of randomized controlled trials. BMC Anesthesiol 22 déc. 2016;16(1):125.

9. Rizzo RRN, Medeiros FC, Pires LG, Pimenta RM, McAuley JH, Jensen MP, et al. Hypnosis Enhances the Effects of Pain Education in Patients With Chronic Nonspecific Low Back Pain: A Randomized Controlled Trial. J Pain. oct 2018;19(10):1103.e1-1103.e9.

10. Sánchez-Jáuregui T, Téllez A, Juárez-García D, García CH, García FE. Clinical Hypnosis and Music In Breast Biopsy:A Randomized Clinical Trial. Am J Clin Hypn janv. 2019;61(3):244-57.

11. Anlló $H$, Herer B, Delignières $A$, Bocahu $Y$, Segundo I, Mach Alingrin $V$, et al. Hypnosis for the Management of Anxiety and Dyspnea in COPD: A Randomized, Sham-Controlled Crossover Trial. Int J Chron Obstruct Pulmon Dis. 2020;15:2609-20.

12. Rousseaux F, Faymonville M-E, Nyssen A-S, Dardenne N, Ledoux D, Massion PB, et al. Can hypnosis and virtual reality reduce anxiety, pain and fatigue among patients who undergo cardiac surgery: a randomised controlled trial. Trials 15 avr. 2020;21(1):330.

13. Liberati A, Altman DG, Tetzlaff J, Mulrow C, Gøtzsche PC, loannidis JPA, et al. The PRISMA statement for reporting systematic reviews and meta-analyses of studies that evaluate health care interventions: explanation and elaboration. PLoS Med. 21 juill 2009;6(7):e1000100.

14. Murad MH, Sultan S, Haffar S, Bazerbachi F. Methodological quality and synthesis of case series and case reports. BMJ Evid Based Med avr. 2018;23(2):60-3.

15. Stang A. Critical evaluation of the Newcastle-Ottawa scale for the assessment of the quality of nonrandomized studies in meta-analyses. Eur J Epidemiol sept. 2010;25(9):603-5.

16. de Morton NA. The PEDro scale is a valid measure of the methodological quality of clinical trials: a demographic study. Aust J Physiother. 2009;55(2):129-33.

17. van Tulder M, Furlan A, Bombardier C, Bouter L. Editorial Board of the Cochrane Collaboration Back Review Group. Updated method guidelines for systematic reviews in the cochrane collaboration back review group. Spine (Phila Pa 1976). 15 juin 2003;28(12):1290-9.

18. Bowen DE. Ventilator weaning through hypnosis. Psychosomatics. 1989;30(4):449-50.

19. Lariccia P, Katz R, Peters J, Atkinson G, Weiss T. Biofeedback and Hypnosis in Weaning from Mechanical Ventilators. Chest. 1985;87(2):267-9.

20. Treggiari-Venzi MM, Suter PM, de Tonnac N, Romand JA. Successful use of hypnosis as an adjunctive therapy for weaning from mechanical ventilation. Anesthesiology mars. 2000;92(3):890- 
2.

21. Acosta F. Weaning the Anxious Ventilator Patient Using Hypnotic Relaxation - Case-Reports. Am J Clin Hypn avr. 1987;29(4):272-80.

\section{Figures}
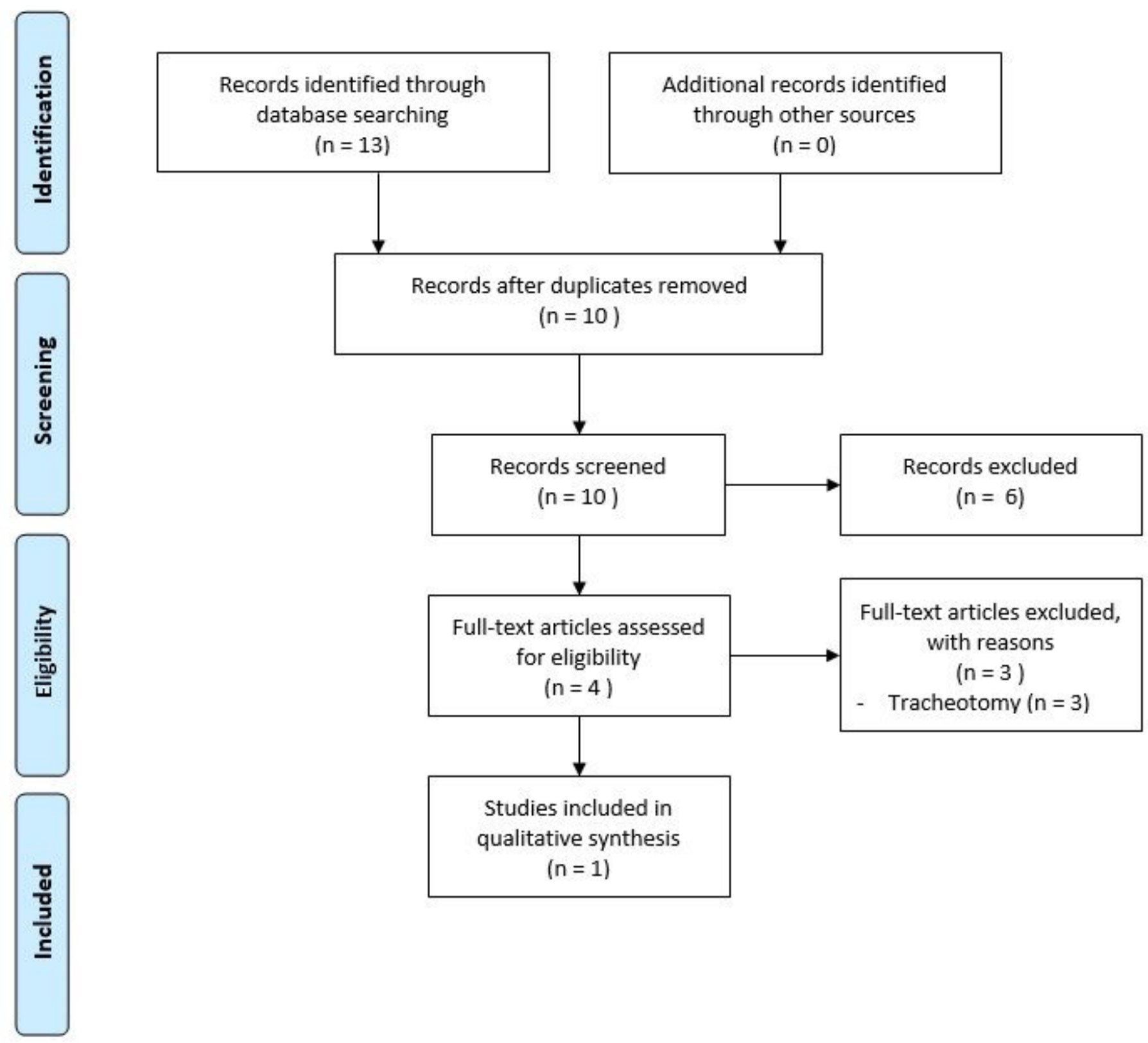

Figure 1

Flow chart of selection studies. This figure shows flow-chart following PRISMA Guidelines. Inclusion and exclusion process are detailed.

\section{Supplementary Files}


This is a list of supplementary files associated with this preprint. Click to download.

- Appendix1SearchStrategy.docx 